\title{
Acknowledgment of Authors
}

The Editors and Staff wish to sincerely thank the following authors who wrote articles for Journal of Oncology Practice in 2010.

Adams, Diana

Adams, Val R.

Alva, Albert A.

Atmar, Jill

Azzoli, Christopher G.

Bach, Peter B.

Baer, Allison R.

Baileys, Kristen

Bainbridge, Daryl

Barbour, Sally Yowell

Barkley, Ronald

Barr, Thomas R.

Bechar, Naftali

Belani, Chandra P.

Bendell, Johanna

Benz, Jr, Edward J.

Beveridge, Roy A.

Beyer, Julie

Blayney, Douglas W.

Blau, Michael L.

Brazil, Kevin

Bridges, Kerry Dune

Britell, Jonathan C.

Brogan, Frank

Bronskill, Susan E.

Brown, Clarence

Bruinooge, Suanna S.

Burris III, Howard A.

Burstein, Harold J.

Cassell, Robert

Celaya, Maria O.

Cherala, Sai

Chua, Wei

Clarke, Stephen J.

Cobos, Everardo

Cohen, Gary

Collyar, Deborah

Coniglio, David

Corrao, Natalie J.

Cox, John V.

Debono, David

Devine, Susan

Doherty, Julia

Donehower, Ross C.

Dreiling, Lyndah

Edge, Stephen B.

Fabian, Carol J.

Feigert, John
Feinberg, Melanie

Ferrell, Betty

Forsyth, Michael

Gagliardi, Anna R.

Gesme, Dean H.

Giaccone, Giuseppe

Gilligan, Timothy D.

Del Giudice, M. Elisabeth

Goldberg, Richard M.

Goldberg-Dey, Judy

Gordon, Alan N.

Gray, Ann

Griggs, Jennifer J.

Grunfeld, Eva

Gruschkus, Stephen K.

Guidi, Teri

Gunderson, Heidi D.

Hammond, M. Elizabeth H.

Hagerty, Karen

Hartgers, Mindy L.

Hayes, Daniel F.

Hayes, Daniel M.

Hendrick, Susan S.

Hennessy, John E.

Hinkel, Jennifer M.

Hoang, Susan

Hodgson, David C.

Hofstatter, Erin W.

Hohneker, John A.

Holcombe, Randall F.

Hollinger, Krista J.

Hoverman, J. Russell

Iacovelli, Lew

Jatoi, Aminah

Jepson, Victoria A.

Johnson, Ali

Kando-Pineda, Carol

Kasper, Michael

Katz, Matthew S.

Kaufman, Peter A.

Keech, Jr, John A.

Kelly, Chelsey A.

Khan, Qamar J.

King, Kristen

Kolodziej, Michael

Kromrey, Jeffrey

Lawson, Amber P.

Legant, Patricia
Levit, Laura

Levy, William

Lin, Jianqing

Look Hong, Nicole J.

Looker, Sherry A.

Luce, Ryan

Lyman, Gary H.

Markman, Maurie

Marks, Alan

Marx, Gavin

Mangu, Pamela B.

Marsland, Thomas

McAneny, Barbara L.

McArthur, Marilyn

McCabe, Mary S.

McCorkle, Michele R.

McWilliams, Robert R.

Miller Murphy, Cynthia

Moffitt, Karen

Moineddin, Rahim

Mukkamala, AppaRao

Mulvey, Therese M.

Naeim, Arash

Neubauer, Marcus A.

Neuss, Michael N.

Nevidjon, Brenda

Nicolaides, Dawn A.

Nunnink, Johannes C.

O'Dwyer, Mary

Ostroff, Joy

Palmer, J. Lynn

Parker, Patricia A.

Paroly, Warren S.

Paszat, Lawrence F.

Peppercorn, Jeffrey M.

Perkel, Sara J.

Philips, Dorothy Green

Phillips, Kathryn A.

Polansky, Maura N.

Prestrud, Ann A.

Provenzano, Anthony

Rees, Judy R.

Reisman, Lonny

Riddle, Bruce

Rieger, Paula

Robbins, Gerald

Robinson, Alan G.

Robinson, William R.
Rolston, Kenneth V. I.

Rosenblatt, Joseph

Rosenzweig, Margaret Quinn

Ross, Alicia C.

Rothermel, Todd

Runowicz, Carolyn D.

Rutstein, Lisa

Santana-Davila, Rafael

Saven, Alan

Schapira, Lidia

Schmit, Marsha

Schumacher, Robert M.

Schwartzberg, Lee $S$.

Schwenn, Molly

Seidenfeld, Jerome

Sessions, Jolynn K.

Shahin, Seta

Smallridge, Robert

Smith, Alison P.

Smith, Emily L.

Smith, Mary Lou

Steinke, Douglas

Stewart, F. Marc

Stewart, Teresa L.

Sullivan, Daniel

Sussman, Jonathan

Sutphin, Stephanie D.

Swiernik, Michael A.

Tanamor, Myra

Temin, Sarah

Thompson, Lisa A.

Tomkins, Julia E.

Towle, Elaine L.

Trosman, Julia R.

Valgus, John

Van Bebber, Stephanie L.

Vandergrift, Jonathan L.

Verrill, Castine

Verschraegen, Claire F.

Waldinger, Marcy B.

Whelan, Tim

Wiseman, Marian

Wolff, Antonio C.

Wood, William A.

Wright, Frances C.

Yasko, Joyce

Zon, Robin T. 